Applied Mathematical Sciences, Vol. 7, 2013, no. 48, 2357 - 2372

HIKARI Ltd, www.m-hikari.com

\title{
Computational Experiments for the Problem of Footstep Planning for Humanoid Robots
}

\author{
Anna Gorbenko \\ Department of Intelligent Systems and Robotics \\ Ural Federal University \\ 620083 Ekaterinburg, Russia \\ gorbenko.ann@gmail.com \\ Vladimir Popov \\ Department of Intelligent Systems and Robotics \\ Ural Federal University \\ 620083 Ekaterinburg, Russia \\ Vladimir.Popov@usu.ru
}

Copyright (c) 2013 Anna Gorbenko and Vladimir Popov. This is an open access article distributed under the Creative Commons Attribution License, which permits unrestricted use, distribution, and reproduction in any medium, provided the original work is properly cited.

\begin{abstract}
It is well known that motion planning problems for humanoid robots are of particular interest. Footstep planning is one of such problems. We describe an approach to solve the footstep planning problem in a free unbounded environment. This approach is based on an explicit reduction from the problem to the satisfiability problem.
\end{abstract}

Keywords: humanoid robot, footstep planning problem, satisfiability

Many different planning problems are among the most rapidly developing areas of modern robotics. In particular, we can mention different localization problems (see e.g. [1]), path and motion planning problems (see e.g. [2], [3]), visual calibration (see e.g. [4]), the problem of sensor placement (see e.g. 
[5] - [8]), allocating complex tasks problems (see e.g. [9] - [12]), automatic generation of visual recognition modules (see e.g. [13] - [15]), the problem of selection of a minimal set of visual landmarks (see e.g. [16] - [18]), selection of partially distinguishable guards (see e.g. [19], [20]), the problem of placement of visual landmarks (see e.g. [21], [22]), systems of robot self-awareness (see e.g. $[23]-[27]$ ), the problem of anticipation of motion (see e.g. [28] - [30]). It should be noted that humanoid robotics has recently made rapid progress. However, there is a rising demand for algorithms useful to improving the autonomy of humanoids. In particular, different planning problems for humanoid robots are of considerable interest (see e.g. [31]). Note that motion planning for humanoid robots requires the solution of many difficult problems. For instance, technical vision problems (see e.g. [32] - [34]), problems of selection of visual landmarks, allocating complex tasks problems, footstep planning (see e.g. [35], [36]). Note that the problem of footstep planning is the problem of motion planning associated with walking motion generation. In this case, we need to compute walking motions that bring the robot from its initial location to a goal location while avoiding obstacles. In this paper, we assume that a robot is walking on a flat ground, free from any obstacle. We consider only mean that only discrete stepping capabilities where only a finite set of possible steps is allowed. Under such conditions, the footstep planning problem can be formulated as the reachability problem. In particular, we can consider in the flat infinite ground a Cartesian coordinate system of two axes $x$ and $y$. We assume that the $x$ axis defines the zero orientation. The configuration of the robot feet in the free environment is completely defined by the position and orientation of the left foot, and the current posture of the feet. So, a sequence of two steps is completely defined by the initial and final posture, and three additional parameters $x, y$, and $\theta$, where $(x, y)$ is the position and $\theta$ is the orientation of the left foot final placement relatively to its initial placement. It is easy to see that we can assume that orientation change is a rational number multiplied by $\pi$. Also, we assume that only a finite set of absolute orientations of the left foot are reachable. A posture is defined by the position $(x, y)$ and orientation $\varphi$ of the right foot relatively to the left foot and by the left foot absolute orientation $\psi$. Therefore, a posture is a quadruple $(u, v, \varphi, \psi)$. Let $\{p[1], \ldots, p[n]\}$ is the set of all the reachable postures. Under such conditions, a configuration of the robot is a posture $p[i]$ and a position of the left foot $(x, y)$. Clearly, we can assume that all the vectors $(p[i], x, y)$ are couples of integer numbers. Similarly, a sequence of two configurations is now completely defined by the initial and final posture and a vector of two parameters. Note that the whole stepping capabilities of the robot can be represented by a 2-counter machine, where $Q=\{p[1], \ldots, p[n]\}$ is the set of states. A configuration of the machine is a triple $(p[i], x, y) \in Q \times Z \times Z$. It is clear that a configuration of the machine exactly corresponds to a configuration of the robot. Each 
allowable sequence of two configurations is a transition $(p[i], x, y, p[j])$. Note that a transition of the machine exactly corresponds to a step of the robot or a change of the robot posture. Reachability Problem (RP): Given a 2counter machine with the set of states $Q$ and the set of transitions $T$ and two configurations $(p[i], x, y)$ and $(p[j], u, v)$. Is there a finite sequence of transitions that goes from $(p[i], x, y)$ to $(p[j], u, v)$ ? Note that RP is $\mathbf{N P}$-complete (see $[37])$. RP can be considered as the fixed distance problem (see e.g. [37]). Let $G$ be a weighted directed multi-graph with set of nodes $V(G)$ and set of edges $E(G)$. We assume that $W(e) \in Z^{k}, e \in E(G)$, is a cost vector.

The Fixed Distance Problem (FDP):

Instance: $p, q \in V(G), D \in Z^{k}$.

QUESTION: Is there a path $e_{1}, \ldots, e_{m}$ such that $p=e_{1}, q=e_{m}, \sum W\left(e_{i}\right)=$ $D$ ?

Encoding different hard problems as instances of different variants of the satisfiability problem and solving them with very efficient satisfiability algorithms has caused considerable interest (see e.g. [38] - [54]). We consider an explicit reduction from FDP to the satisfiability problem.

We consider the following nondeterministic algorithm (sf. [55]). We add into $E(G)$ a new edge $f$ from $q$ to $p$ such that $W(f)=0$. We guess a subgraph $H$ of $G$ such that $H$ contains $f$ and forms a strongly connected component. We consider the following ILP instance. There is one variable $c[e]$ for every $e \in E(H)$ where $E(H)$ is the set of edges of $H$. There are no other variables. The constraints are as follows. Let $c[f]=1$. Let $c[e] \geq 1$, for all $e \in E(H)$ such that $e \neq f$. Let $V(H)$ be the set of nodes of $H$. Let in $(r)$ consists of the edges entering $r$ in $H$. Let out $(r)$ consists of the edges $r$ exiting in $H$. For all $r \in V(H)$, we assume that $\sum_{e \in i n(r)} c[e]=\sum_{e \in o u t(r)} c[e]$. Let $D=$ $\left(d_{1}, d_{2}, \ldots, d_{k}\right), W(e)=\left(w_{1}(e), w_{2}(e), \ldots, w_{k}(e)\right)$. For any $1 \leq i \leq k$, we assume that $d_{i}=\sum_{e \in E(H)} c[e] w_{i}(e)$. There is a solution to the ILP instance if and only if there is a path $e_{1}, e_{2}, \ldots, e_{m}$ from $p$ to $q$ such that $\sum_{i=1}^{m} W\left(e_{i}\right)=D$ (see [55]). Let $\delta=\max \left\{\max _{1 \leq i \leq k}\left\{\left|d_{i}\right|\right\}, \max _{e \in E(H)}\left\{\max _{1 \leq i \leq k}\left\{\left|w_{i}(e)\right|\right\}\right\}\right\}$.

Note that if there is a solution to the ILP instance, then for any $e \in E(H)$ we can assume that $c[e] \leq(|E(G)|+1)(\delta(k+|V(G)|+1))^{2(k+|V(G)|+1)+1}$ (sf. [56]). We assume that $e$ exiting $i n(e)$ in $H$ and entering out $(e)$ in $H$. If $d_{i} \geq 0$, then we assume that $d_{i}^{+}=d_{i}, d_{i}^{-}=0$. If $d_{i}<0$, then $d_{i}^{+}=0, d_{i}^{-}=\left|d_{i}\right|$. Let

$$
\begin{gathered}
V(G)=\left\{p_{1}, p_{2}, \ldots, p_{|V(G)|}\right\}, p_{1}=p, p_{2}=q, \\
E(G) \cup\{f\}=\left\{e_{1}, e_{2}, \ldots, e_{|E(G)|+1}\right\}, e_{1}=f, \\
M=\left\lceil\log _{2}\left((|E(G)|+1)(\delta(k+|V(G)|+1))^{2(k+|V(G)|+1)+1}\right)\right\rceil, \\
c\left[e_{i}\right]=\sum_{j=0}^{M} z[i, j] 2^{j}, d_{i}^{+}=\sum_{j=0}^{M} d^{+}[i, j] 2^{j}, d_{i}^{-}=\sum_{j=0}^{M} d^{-}[i, j] 2^{j}, \\
\varphi[1]=\wedge_{1 \leq i \leq|V(G)|} \wedge_{1 \leq j \leq|V(G)|}(\neg x[i] \vee \neg x[j] \vee y[1, i, j, 1]),
\end{gathered}
$$




$$
\begin{aligned}
& \varphi[2]=\wedge_{1 \leq i \leq|V(G)|} \wedge_{1 \leq j \leq|V(G)|} \wedge_{1 \leq s \leq|E(G)|}(y[1, i, j, s] \vee \neg y[1, i, j, s+1]),
\end{aligned}
$$

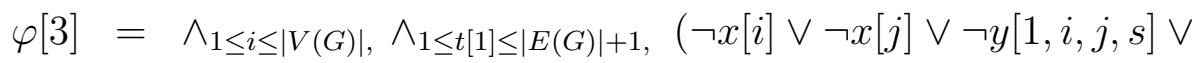

$$
\begin{aligned}
& 1 \leq j \leq|V(G)|, \quad 1 \leq t[2] \leq|E(G)|+1, \\
& 1 \leq s \leq|E(G)| \quad \text { out }\left(e_{t[1]}\right) \neq i n\left(e_{t[2]}\right) \\
& \neg y[1, i, j, s+1] \vee \neg y[2, i, j, s, t[1]] \vee \neg y[2, i, j, s+1, t[2]]),
\end{aligned}
$$

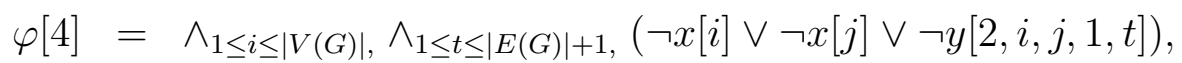

$$
\begin{aligned}
& 1 \leq j \leq|V(G)| \quad p_{i} \neq i n\left(e_{t}\right) \\
& \varphi[5]=\wedge_{1 \leq i \leq|V(G)|} \wedge_{1 \leq j \leq|V(G)|} \wedge_{1 \leq s \leq|E(G)|} \wedge_{1 \leq t \leq|E(G)|+1, p_{j} \neq \text { out }\left(e_{t}\right)}(\neg x[i] \vee \\
& \neg x[j] \vee \neg y[1, i, j, s] \vee y[1, i, j, s+1] \vee \neg y[2, i, j, s, t]), \\
& \varphi[6]=\wedge_{1 \leq i \leq|V(G)|} \wedge_{1 \leq j \leq|V(G)|} \wedge_{1 \leq t \leq|E(G)|+1, p_{j} \neq \text { out }\left(e_{t}\right)}(\neg x[i] \vee \\
& \neg x[j] \vee \neg y[1, i, j,|E(G)|+1] \vee \neg y[2, i, j,|E(G)|+1, t]), \\
& \varphi[7]=\wedge_{1 \leq i \leq|V(G)|} \wedge_{1 \leq j \leq|V(G)|} \wedge_{1 \leq s \leq|E(G)|+1} \vee_{1 \leq t \leq|E(G)|+1} y[2, i, j, s, t],
\end{aligned}
$$

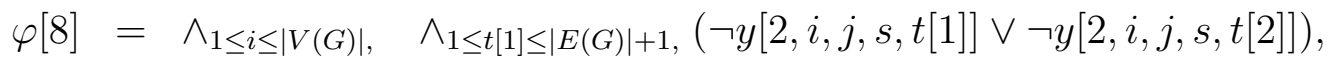

$$
\begin{aligned}
& 1 \leq j \leq|V(G)|, \quad 1 \leq t[2] \leq|E(G)|+1, \\
& 1 \leq s \leq|E(G)|+1 \quad t[1] \neq t[2] \\
& \varphi[9]=z[1,0] \\
& \varphi[10]=\wedge_{1 \leq i \leq M} \neg z[1, i] \text {, } \\
& \varphi[11]=\wedge_{2 \leq i \leq|E(G)|+1} \vee_{0 \leq j \leq M} z[i, j], \\
& \varphi[12]=\wedge_{1 \leq i \leq|V(G)|} \wedge_{0 \leq j \leq M} \neg u[1, i, 0, j], \\
& \varphi[13]=\wedge_{1 \leq i \leq|V(G)|} \wedge_{1 \leq j \leq|E(G)|+1} \neg u[2, i, j, 0] \text {, } \\
& \varphi[14]=\wedge_{1 \leq i \leq|V(G)|} \wedge_{1 \leq j \leq|E(G)|+1, e_{j} \in i n\left(p_{i}\right)} \wedge_{0 \leq s \leq M}(\neg u[1, i, j-1, s] \wedge \\
& \neg z[j, s] \wedge \neg u[2, i, j, s]) \rightarrow \neg u[1, i, j, s], \\
& \varphi[15]=\wedge_{1 \leq i \leq|V(G)|} \wedge_{1 \leq j \leq|E(G)|+1, e_{j} \in \operatorname{in}\left(p_{i}\right)} \wedge_{0 \leq s \leq M}(\neg u[1, i, j-1, s] \wedge \\
& \neg z[j, s] \wedge \neg u[2, i, j, s]) \rightarrow \neg u[2, i, j, s+1], \\
& \varphi[16]=\wedge_{1 \leq i \leq|V(G)|} \wedge_{1 \leq j \leq|E(G)|+1, e_{j} \in \operatorname{in}\left(p_{i}\right)} \wedge_{0 \leq s \leq M}(u[1, i, j-1, s] \wedge \\
& \neg z[j, s] \wedge \neg u[2, i, j, s]) \rightarrow u[1, i, j, s], \\
& \varphi[17]=\wedge_{1 \leq i \leq|V(G)|} \wedge_{1 \leq j \leq|E(G)|+1, e_{j} \in \operatorname{in}\left(p_{i}\right)} \wedge_{0 \leq s \leq M}(u[1, i, j-1, s] \wedge \\
& \neg z[j, s] \wedge \neg u[2, i, j, s]) \rightarrow \neg u[2, i, j, s+1], \\
& \varphi[18]=\wedge_{1 \leq i \leq|V(G)|} \wedge_{1 \leq j \leq|E(G)|+1, e_{j} \in \operatorname{in}\left(p_{i}\right)} \wedge_{0 \leq s \leq M}(\neg u[1, i, j-1, s] \wedge \\
& z[j, s] \wedge \neg u[2, i, j, s]) \rightarrow u[1, i, j, s], \\
& \varphi[19]=\wedge_{1 \leq i \leq|V(G)|} \wedge_{1 \leq j \leq|E(G)|+1, e_{j} \in \operatorname{in}\left(p_{i}\right)} \wedge_{0 \leq s \leq M}(\neg u[1, i, j-1, s] \wedge \\
& z[j, s] \wedge \neg u[2, i, j, s]) \rightarrow \neg u[2, i, j, s+1], \\
& \varphi[20]=\wedge_{1 \leq i \leq|V(G)|} \wedge_{1 \leq j \leq|E(G)|+1, e_{j} \in \operatorname{in}\left(p_{i}\right)} \wedge_{0 \leq s \leq M}(\neg u[1, i, j-1, s] \wedge \\
& \neg z[j, s] \wedge u[2, i, j, s]) \rightarrow u[1, i, j, s], \\
& \varphi[21]=\wedge_{1 \leq i \leq|V(G)|} \wedge_{1 \leq j \leq|E(G)|+1, e_{j} \in \operatorname{in}\left(p_{i}\right)} \wedge_{0 \leq s \leq M}(\neg u[1, i, j-1, s] \wedge \\
& \neg z[j, s] \wedge u[2, i, j, s]) \rightarrow \neg u[2, i, j, s+1],
\end{aligned}
$$




$$
\begin{aligned}
& \varphi[22]=\wedge_{1 \leq i \leq|V(G)|} \wedge_{1 \leq j \leq|E(G)|+1, e_{j} \in \operatorname{in}\left(p_{i}\right)} \wedge_{0 \leq s \leq M}(u[1, i, j-1, s] \wedge \\
& z[j, s] \wedge \neg u[2, i, j, s]) \rightarrow \neg u[1, i, j, s], \\
& \varphi[23]=\wedge_{1 \leq i \leq|V(G)|} \wedge_{1 \leq j \leq|E(G)|+1, e_{j} \in i n\left(p_{i}\right)} \wedge_{0 \leq s \leq M}(u[1, i, j-1, s] \wedge \\
& z[j, s] \wedge \neg u[2, i, j, s]) \rightarrow u[2, i, j, s+1], \\
& \varphi[24]=\wedge_{1 \leq i \leq|V(G)|} \wedge_{1 \leq j \leq|E(G)|+1, e_{j} \in \operatorname{in}\left(p_{i}\right)} \wedge_{0 \leq s \leq M}(u[1, i, j-1, s] \wedge \\
& \neg z[j, s] \wedge u[2, i, j, s]) \rightarrow \neg u[1, i, j, s], \\
& \varphi[25]=\wedge_{1 \leq i \leq|V(G)|} \wedge_{1 \leq j \leq|E(G)|+1, e_{j} \in i n\left(p_{i}\right)} \wedge_{0 \leq s \leq M}(u[1, i, j-1, s] \wedge \\
& \neg z[j, s] \wedge u[2, i, j, s]) \rightarrow u[2, i, j, s+1], \\
& \varphi[26]=\wedge_{1 \leq i \leq|V(G)|} \wedge_{1 \leq j \leq|E(G)|+1, e_{j} \in i n\left(p_{i}\right)} \wedge_{0 \leq s \leq M}(\neg u[1, i, j-1, s] \wedge \\
& z[j, s] \wedge u[2, i, j, s]) \rightarrow \neg u[1, i, j, s], \\
& \varphi[27]=\wedge_{1 \leq i \leq|V(G)|} \wedge_{1 \leq j \leq|E(G)|+1, e_{j} \in \operatorname{in}\left(p_{i}\right)} \wedge_{0 \leq s \leq M}(\neg u[1, i, j-1, s] \wedge \\
& z[j, s] \wedge u[2, i, j, s]) \rightarrow u[2, i, j, s+1], \\
& \varphi[28]=\wedge_{1 \leq i \leq|V(G)|} \wedge_{1 \leq j \leq|E(G)|+1, e_{j} \in \operatorname{in}\left(p_{i}\right)} \wedge_{0 \leq s \leq M}(u[1, i, j-1, s] \wedge \\
& z[j, s] \wedge u[2, i, j, s]) \rightarrow u[1, i, j, s], \\
& \varphi[29]=\wedge_{1 \leq i \leq|V(G)|} \wedge_{1 \leq j \leq|E(G)|+1, e_{j} \in \operatorname{in}\left(p_{i}\right)} \wedge_{0 \leq s \leq M}(u[1, i, j-1, s] \wedge \\
& z[j, s] \wedge u[2, i, j, s]) \rightarrow u[2, i, j, s+1], \\
& \varphi[30]=\wedge_{1 \leq i \leq|V(G)|, 1 \leq j \leq|E(G)|+1, e_{j} \notin i n\left(p_{i}\right), 0 \leq s \leq M} u[1, i, j-1, s]=u[1, i, j, s], \\
& \varphi[31]=\wedge_{1 \leq i \leq|V(G)|} \wedge_{0 \leq j \leq M} \neg u[3, i, 0, j] \text {, } \\
& \varphi[32]=\wedge_{1 \leq i \leq|V(G)|} \wedge_{1 \leq j \leq|E(G)|+1} \neg u[4, i, j, 0] \text {, } \\
& \varphi[33]=\wedge_{1 \leq i \leq|V(G)|} \wedge_{1 \leq j \leq|E(G)|+1, e_{j} \in \text { out }\left(p_{i}\right)} \wedge_{0 \leq s \leq M}(\neg u[3, i, j-1, s] \wedge \\
& \neg z[j, s] \wedge \neg u[4, i, j, s]) \rightarrow \neg u[3, i, j, s], \\
& \varphi[34]=\wedge_{1 \leq i \leq|V(G)|} \wedge_{1 \leq j \leq|E(G)|+1, e_{j} \in \text { out }\left(p_{i}\right)} \wedge_{0 \leq s \leq M}(\neg u[3, i, j-1, s] \wedge \\
& \neg z[j, s] \wedge \neg u[4, i, j, s]) \rightarrow \neg u[4, i, j, s+1], \\
& \varphi[35]=\wedge_{1 \leq i \leq|V(G)|} \wedge_{1 \leq j \leq|E(G)|+1, e_{j} \in \text { out }\left(p_{i}\right)} \wedge_{0 \leq s \leq M}(u[3, i, j-1, s] \wedge \\
& \neg z[j, s] \wedge \neg u[4, i, j, s]) \rightarrow u[3, i, j, s], \\
& \varphi[36]=\wedge_{1 \leq i \leq|V(G)|} \wedge_{1 \leq j \leq|E(G)|+1, e_{j} \in \text { out }\left(p_{i}\right)} \wedge_{0 \leq s \leq M}(u[3, i, j-1, s] \wedge \\
& \neg z[j, s] \wedge \neg u[4, i, j, s]) \rightarrow \neg u[4, i, j, s+1], \\
& \varphi[37]=\wedge_{1 \leq i \leq|V(G)|} \wedge_{1 \leq j \leq|E(G)|+1, e_{j} \in \text { out }\left(p_{i}\right)} \wedge_{0 \leq s \leq M}(\neg u[3, i, j-1, s] \wedge \\
& z[j, s] \wedge \neg u[4, i, j, s]) \rightarrow u[3, i, j, s], \\
& \varphi[38]=\wedge_{1 \leq i \leq|V(G)|} \wedge_{1 \leq j \leq|E(G)|+1, e_{j} \in \text { out }\left(p_{i}\right)} \wedge_{0 \leq s \leq M}(\neg u[3, i, j-1, s] \wedge \\
& z[j, s] \wedge \neg u[4, i, j, s]) \rightarrow \neg u[4, i, j, s+1], \\
& \varphi[39]=\wedge_{1 \leq i \leq|V(G)|} \wedge_{1 \leq j \leq|E(G)|+1, e_{j} \in \text { out }\left(p_{i}\right)} \wedge_{0 \leq s \leq M}(\neg u[3, i, j-1, s] \wedge \\
& \neg z[j, s] \wedge u[4, i, j, s]) \rightarrow u[3, i, j, s], \\
& \varphi[40]=\wedge_{1 \leq i \leq|V(G)|} \wedge_{1 \leq j \leq|E(G)|+1, e_{j} \in \text { out }\left(p_{i}\right)} \wedge_{0 \leq s \leq M}(\neg u[3, i, j-1, s] \wedge \\
& \neg z[j, s] \wedge u[4, i, j, s]) \rightarrow \neg u[4, i, j, s+1],
\end{aligned}
$$




$$
\begin{aligned}
& \varphi[41]=\wedge_{1 \leq i \leq|V(G)|} \wedge_{1 \leq j \leq|E(G)|+1, e_{j} \in \text { out }\left(p_{i}\right)} \wedge_{0 \leq s \leq M}(u[3, i, j-1, s] \wedge \\
& z[j, s] \wedge \neg u[4, i, j, s]) \rightarrow \neg u[3, i, j, s] \\
& \varphi[42]=\wedge_{1 \leq i \leq|V(G)|} \wedge_{1 \leq j \leq|E(G)|+1, e_{j} \in \text { out }\left(p_{i}\right)} \wedge_{0 \leq s \leq M}(u[3, i, j-1, s] \wedge \\
& z[j, s] \wedge \neg u[4, i, j, s]) \rightarrow u[4, i, j, s+1] \\
& \varphi[43]=\wedge_{1 \leq i \leq|V(G)|} \wedge_{1 \leq j \leq|E(G)|+1, e_{j} \in \text { out }\left(p_{i}\right)} \wedge_{0 \leq s \leq M}(u[3, i, j-1, s] \wedge \\
& \neg z[j, s] \wedge u[4, i, j, s]) \rightarrow \neg u[3, i, j, s] \\
& \varphi[44]=\wedge_{1 \leq i \leq|V(G)|} \wedge_{1 \leq j \leq|E(G)|+1, e_{j} \in \text { out }\left(p_{i}\right)} \wedge_{0 \leq s \leq M}(u[3, i, j-1, s] \wedge \\
& \neg z[j, s] \wedge u[4, i, j, s]) \rightarrow u[4, i, j, s+1], \\
& \varphi[45]=\wedge_{1 \leq i \leq|V(G)|} \wedge_{1 \leq j \leq|E(G)|+1, e_{j} \in \text { out }\left(p_{i}\right)} \wedge_{0 \leq s \leq M}(\neg u[3, i, j-1, s] \wedge \\
& z[j, s] \wedge u[4, i, j, s]) \rightarrow \neg u[3, i, j, s], \\
& \varphi[46]=\wedge_{1 \leq i \leq|V(G)|} \wedge_{1 \leq j \leq|E(G)|+1, e_{j} \in \text { out }\left(p_{i}\right)} \wedge_{0 \leq s \leq M}(\neg u[3, i, j-1, s] \wedge \\
& z[j, s] \wedge u[4, i, j, s]) \rightarrow u[4, i, j, s+1], \\
& \varphi[47]=\wedge_{1 \leq i \leq|V(G)|} \wedge_{1 \leq j \leq|E(G)|+1, e_{j} \in \text { out }\left(p_{i}\right)} \wedge_{0 \leq s \leq M}(u[3, i, j-1, s] \wedge \\
& z[j, s] \wedge u[4, i, j, s]) \rightarrow u[3, i, j, s], \\
& \varphi[48]=\wedge_{1 \leq i \leq|V(G)|} \wedge_{1 \leq j \leq|E(G)|+1, e_{j} \in \text { out }\left(p_{i}\right)} \wedge_{0 \leq s \leq M}(u[3, i, j-1, s] \wedge \\
& z[j, s] \wedge u[4, i, j, s]) \rightarrow u[4, i, j, s+1], \\
& \varphi[49]=\wedge_{1 \leq i \leq|V(G)|, 1 \leq j \leq|E(G)|+1, e_{j} \notin \text { out }\left(p_{i}\right), 0 \leq s \leq M} u[3, i, j-1, s]=u[3, i, j, s], \\
& \varphi[50]=\wedge_{1 \leq i \leq|V(G)|} \wedge_{0 \leq s \leq M} \neg x[i] \vee \\
& u[1, i,|E(G)|+1, s]=u[3, i,|E(G)|+1, s], \\
& \varphi[51]=\wedge_{1 \leq i \leq k} \wedge_{1 \leq j \leq|E(G)|+1} \wedge_{0 \leq s \leq M} \neg u[5, i, j, 0, s] \text {, } \\
& \varphi[52]=\wedge_{1 \leq i \leq k} \wedge_{1 \leq j \leq|E(G)|+1} \wedge_{0 \leq t \leq\left|w_{i}\left(e_{j}\right)\right| \neg u[6, i, j, t, 0]}, \\
& \varphi[53]=\wedge_{1 \leq i \leq k} \wedge_{1 \leq j \leq|E(G)|+1} \wedge_{1 \leq t \leq\left|w_{i}\left(e_{j}\right)\right|} \wedge_{0 \leq s \leq M}(\neg u[5, i, j, t-1, s] \wedge \\
& \neg z[j, s] \wedge \neg u[6, i, j, t, s]) \rightarrow \neg u[5, i, j, t, s], \\
& \varphi[54]=\wedge_{1 \leq i \leq k} \wedge_{1 \leq j \leq|E(G)|+1} \wedge_{1 \leq t \leq\left|w_{i}\left(e_{j}\right)\right|} \wedge_{0 \leq s \leq M}(\neg u[5, i, j, t-1, s] \wedge \\
& \neg z[j, s] \wedge \neg u[6, i, j, t, s]) \rightarrow \neg u[6, i, j, t, s+1], \\
& \varphi[55]=\wedge_{1 \leq i \leq k} \wedge_{1 \leq j \leq|E(G)|+1} \wedge_{1 \leq t \leq\left|w_{i}\left(e_{j}\right)\right|} \wedge_{0 \leq s \leq M}(\neg u[5, i, j, t-1, s] \wedge \\
& \neg z[j, s] \wedge u[6, i, j, t, s]) \rightarrow u[5, i, j, t, s], \\
& \varphi[56]=\wedge_{1 \leq i \leq k} \wedge_{1 \leq j \leq|E(G)|+1} \wedge_{1 \leq t \leq\left|w_{i}\left(e_{j}\right)\right|} \wedge_{0 \leq s \leq M}(\neg u[5, i, j, t-1, s] \wedge \\
& \neg z[j, s] \wedge u[6, i, j, t, s]) \rightarrow \neg u[6, i, j, t, s+1], \\
& \varphi[57]=\wedge_{1 \leq i \leq k} \wedge_{1 \leq j \leq|E(G)|+1} \wedge_{1 \leq t \leq\left|w_{i}\left(e_{j}\right)\right|} \wedge_{0 \leq s \leq M}(\neg u[5, i, j, t-1, s] \wedge \\
& z[j, s] \wedge \neg u[6, i, j, t, s]) \rightarrow u[5, i, j, t, s], \\
& \varphi[58]=\wedge_{1 \leq i \leq k} \wedge_{1 \leq j \leq|E(G)|+1} \wedge_{1 \leq t \leq\left|w_{i}\left(e_{j}\right)\right|} \wedge_{0 \leq s \leq M}(\neg u[5, i, j, t-1, s] \wedge \\
& z[j, s] \wedge \neg u[6, i, j, t, s]) \rightarrow \neg u[6, i, j, t, s+1], \\
& \varphi[59]=\wedge_{1 \leq i \leq k} \wedge_{1 \leq j \leq|E(G)|+1} \wedge_{1 \leq t \leq\left|w_{i}\left(e_{j}\right)\right|} \wedge_{0 \leq s \leq M}(u[5, i, j, t-1, s] \wedge \\
& \neg z[j, s] \wedge \neg u[6, i, j, t, s]) \rightarrow u[5, i, j, t, s],
\end{aligned}
$$




$$
\begin{aligned}
& \varphi[60]=\wedge_{1 \leq i \leq k} \wedge_{1 \leq j \leq|E(G)|+1} \wedge_{1 \leq t \leq\left|w_{i}\left(e_{j}\right)\right|} \wedge_{0 \leq s \leq M}(u[5, i, j, t-1, s] \wedge \\
& \neg z[j, s] \wedge \neg u[6, i, j, t, s]) \rightarrow \neg u[6, i, j, t, s+1], \\
& \varphi[61]=\wedge_{1 \leq i \leq k} \wedge_{1 \leq j \leq|E(G)|+1} \wedge_{1 \leq t \leq\left|w_{i}\left(e_{j}\right)\right|} \wedge_{0 \leq s \leq M}(u[5, i, j, t-1, s] \wedge \\
& z[j, s] \wedge \neg u[6, i, j, t, s]) \rightarrow \neg u[5, i, j, t, s], \\
& \varphi[62]=\wedge_{1 \leq i \leq k} \wedge_{1 \leq j \leq|E(G)|+1} \wedge_{1 \leq t \leq\left|w_{i}\left(e_{j}\right)\right|} \wedge_{0 \leq s \leq M}(u[5, i, j, t-1, s] \wedge \\
& z[j, s] \wedge \neg u[6, i, j, t, s]) \rightarrow u[6, i, j, t, s+1], \\
& \varphi[63]=\wedge_{1 \leq i \leq k} \wedge_{1 \leq j \leq|E(G)|+1} \wedge_{1 \leq t \leq\left|w_{i}\left(e_{j}\right)\right|} \wedge_{0 \leq s \leq M}(u[5, i, j, t-1, s] \wedge \\
& \neg z[j, s] \wedge u[6, i, j, t, s]) \rightarrow \neg u[5, i, j, t, s], \\
& \varphi[64]=\wedge_{1 \leq i \leq k} \wedge_{1 \leq j \leq|E(G)|+1} \wedge_{1 \leq t \leq\left|w_{i}\left(e_{j}\right)\right|} \wedge_{0 \leq s \leq M}(u[5, i, j, t-1, s] \wedge \\
& \neg z[j, s] \wedge u[6, i, j, t, s]) \rightarrow u[6, i, j, t, s+1], \\
& \varphi[65]=\wedge_{1 \leq i \leq k} \wedge_{1 \leq j \leq|E(G)|+1} \wedge_{1 \leq t \leq\left|w_{i}\left(e_{j}\right)\right|} \wedge_{0 \leq s \leq M}(\neg u[5, i, j, t-1, s] \wedge \\
& z[j, s] \wedge u[6, i, j, t, s]) \rightarrow \neg u[5, i, j, t, s], \\
& \varphi[66]=\wedge_{1 \leq i \leq k} \wedge_{1 \leq j \leq|E(G)|+1} \wedge_{1 \leq t \leq\left|w_{i}\left(e_{j}\right)\right|} \wedge_{0 \leq s \leq M}(\neg u[5, i, j, t-1, s] \wedge \\
& z[j, s] \wedge u[6, i, j, t, s]) \rightarrow u[6, i, j, t, s+1],
\end{aligned}
$$

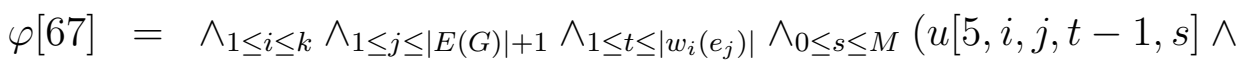

$$
\begin{aligned}
& z[j, s] \wedge u[6, i, j, t, s]) \rightarrow u[5, i, j, t, s], \\
& \varphi[68]=\wedge_{1 \leq i \leq k} \wedge_{1 \leq j \leq|E(G)|+1} \wedge_{1 \leq t \leq\left|w_{i}\left(e_{j}\right)\right|} \wedge_{0 \leq s \leq M}(u[5, i, j, t-1, s] \wedge \\
& z[j, s] \wedge u[6, i, j, t, s]) \rightarrow u[6, i, j, t, s+1], \\
& \varphi[69]=\wedge_{1 \leq i \leq k} \wedge_{0 \leq s \leq M} \neg u[7, i, 0, s] \text {, } \\
& \varphi[70]=\wedge_{1 \leq i \leq k} \wedge_{1 \leq j \leq|E(G)|+1} \neg u[8, i, j, 0] \text {, } \\
& \varphi[71]=\wedge_{1 \leq i \leq k} \wedge_{1 \leq j \leq|E(G)|+1, w_{i}\left(e_{j}\right) \geq 0} \wedge_{0 \leq s \leq M}(\neg u[7, i, j-1, s] \wedge \\
& \left.\neg u\left[5, i, j,\left|w_{i}\left(e_{j}\right)\right|, s\right] \wedge \neg u[8, i, j, s]\right) \rightarrow \neg u[7, i, j, s], \\
& \varphi[72]=\wedge_{1 \leq i \leq k} \wedge_{1 \leq j \leq|E(G)|+1, w_{i}\left(e_{j}\right) \geq 0} \wedge_{0 \leq s \leq M}(\neg u[7, i, j-1, s] \wedge \\
& \left.\neg u\left[5, i, j,\left|w_{i}\left(e_{j}\right)\right|, s\right] \wedge \neg u[8, i, j, s]\right) \rightarrow \neg u[8, i, j, s+1], \\
& \varphi[73]=\wedge_{1 \leq i \leq k} \wedge_{1 \leq j \leq|E(G)|+1, w_{i}\left(e_{j}\right) \geq 0} \wedge_{0 \leq s \leq M}(u[7, i, j-1, s] \wedge \\
& \left.\neg u\left[5, i, j,\left|w_{i}\left(e_{j}\right)\right|, s\right] \wedge \neg u[8, i, j, s]\right) \rightarrow u[7, i, j, s], \\
& \varphi[74]=\wedge_{1 \leq i \leq k} \wedge_{1 \leq j \leq|E(G)|+1, w_{i}\left(e_{j}\right) \geq 0} \wedge_{0 \leq s \leq M}(u[7, i, j-1, s] \wedge \\
& \left.\neg u\left[5, i, j,\left|w_{i}\left(e_{j}\right)\right|, s\right] \wedge \neg u[8, i, j, s]\right) \rightarrow \neg u[8, i, j, s+1], \\
& \varphi[75]=\wedge_{1 \leq i \leq k} \wedge_{1 \leq j \leq|E(G)|+1, w_{i}\left(e_{j}\right) \geq 0} \wedge_{0 \leq s \leq M}(\neg u[7, i, j-1, s] \wedge \\
& \left.u\left[5, i, j,\left|w_{i}\left(e_{j}\right)\right|, s\right] \wedge \neg u[8, i, j, s]\right) \rightarrow u[7, i, j, s], \\
& \varphi[76]=\wedge_{1 \leq i \leq k} \wedge_{1 \leq j \leq|E(G)|+1, w_{i}\left(e_{j}\right) \geq 0} \wedge_{0 \leq s \leq M}(\neg u[7, i, j-1, s] \wedge \\
& \left.u\left[5, i, j,\left|w_{i}\left(e_{j}\right)\right|, s\right] \wedge \neg u[8, i, j, s]\right) \rightarrow \neg u[8, i, j, s+1], \\
& \varphi[77]=\wedge_{1 \leq i \leq k} \wedge_{1 \leq j \leq|E(G)|+1, w_{i}\left(e_{j}\right) \geq 0} \wedge_{0 \leq s \leq M}(\neg u[7, i, j-1, s] \wedge \\
& \left.\neg u\left[5, i, j,\left|w_{i}\left(e_{j}\right)\right|, s\right] \wedge u[8, i, j, s]\right) \rightarrow u[7, i, j, s], \\
& \varphi[78]=\wedge_{1 \leq i \leq k} \wedge_{1 \leq j \leq|E(G)|+1, w_{i}\left(e_{j}\right) \geq 0} \wedge_{0 \leq s \leq M}(\neg u[7, i, j-1, s] \wedge
\end{aligned}
$$




$$
\begin{aligned}
& \left.\neg u\left[5, i, j,\left|w_{i}\left(e_{j}\right)\right|, s\right] \wedge u[8, i, j, s]\right) \rightarrow \neg u[8, i, j, s+1], \\
& \varphi[79]=\wedge_{1 \leq i \leq k} \wedge_{1 \leq j \leq|E(G)|+1, w_{i}\left(e_{j}\right) \geq 0} \wedge_{0 \leq s \leq M}(u[7, i, j-1, s] \wedge \\
& \left.u\left[5, i, j,\left|w_{i}\left(e_{j}\right)\right|, s\right] \wedge \neg u[8, i, j, s]\right) \rightarrow \neg u[7, i, j, s], \\
& \varphi[80]=\wedge_{1 \leq i \leq k} \wedge_{1 \leq j \leq|E(G)|+1, w_{i}\left(e_{j}\right) \geq 0} \wedge_{0 \leq s \leq M}(u[7, i, j-1, s] \wedge \\
& \left.u\left[5, i, j,\left|w_{i}\left(e_{j}\right)\right|, s\right] \wedge \neg u[8, i, j, s]\right) \rightarrow u[8, i, j, s+1], \\
& \varphi[81]=\wedge_{1 \leq i \leq k} \wedge_{1 \leq j \leq|E(G)|+1, w_{i}\left(e_{j}\right) \geq 0} \wedge_{0 \leq s \leq M}(u[7, i, j-1, s] \wedge \\
& \left.\neg u\left[5, i, j,\left|w_{i}\left(e_{j}\right)\right|, s\right] \wedge u[8, i, j, s]\right) \rightarrow \neg u[7, i, j, s], \\
& \varphi[82]=\wedge_{1 \leq i \leq k} \wedge_{1 \leq j \leq|E(G)|+1, w_{i}\left(e_{j}\right) \geq 0} \wedge_{0 \leq s \leq M}(u[7, i, j-1, s] \wedge \\
& \left.\neg u\left[5, i, j,\left|w_{i}\left(e_{j}\right)\right|, s\right] \wedge u[8, i, j, s]\right) \rightarrow u[8, i, j, s+1], \\
& \varphi[83]=\wedge_{1 \leq i \leq k} \wedge_{1 \leq j \leq|E(G)|+1, w_{i}\left(e_{j}\right) \geq 0} \wedge_{0 \leq s \leq M}(\neg u[7, i, j-1, s] \wedge \\
& \left.u\left[5, i, j,\left|w_{i}\left(e_{j}\right)\right|, s\right] \wedge u[8, i, j, s]\right) \rightarrow \neg u[7, i, j, s], \\
& \varphi[84]=\wedge_{1 \leq i \leq k} \wedge_{1 \leq j \leq|E(G)|+1, w_{i}\left(e_{j}\right) \geq 0} \wedge_{0 \leq s \leq M}(\neg u[7, i, j-1, s] \wedge \\
& \left.u\left[5, i, j,\left|w_{i}\left(e_{j}\right)\right|, s\right] \wedge u[8, i, j, s]\right) \rightarrow u[8, i, j, s+1], \\
& \varphi[85]=\wedge_{1 \leq i \leq k} \wedge_{1 \leq j \leq|E(G)|+1, w_{i}\left(e_{j}\right) \geq 0} \wedge_{0 \leq s \leq M}(u[7, i, j-1, s] \wedge \\
& \left.u\left[5, i, j,\left|w_{i}\left(e_{j}\right)\right|, s\right] \wedge u[8, i, j, s]\right) \rightarrow u[7, i, j, s], \\
& \varphi[86]=\wedge_{1 \leq i \leq k} \wedge_{1 \leq j \leq|E(G)|+1, w_{i}\left(e_{j}\right) \geq 0} \wedge_{0 \leq s \leq M}(u[7, i, j-1, s] \wedge \\
& \left.u\left[5, i, j,\left|w_{i}\left(e_{j}\right)\right|, s\right] \wedge u[8, i, j, s]\right) \rightarrow u[8, i, j, s+1], \\
& \varphi[87]=\wedge_{1 \leq i \leq k} \wedge_{1 \leq j \leq|E(G)|+1, w_{i}\left(e_{j}\right)<0} \wedge_{0 \leq s \leq M} u[7, i, j-1, s]=u[7, i, j, s], \\
& \varphi[88]=\wedge_{1 \leq i \leq k} \wedge_{0 \leq s \leq M} \neg u[9, i, 0, s] \text {, } \\
& \varphi[89]=\wedge_{1 \leq i \leq k} \wedge_{1 \leq j \leq|E(G)|+1} \neg u[10, i, j, 0], \\
& \varphi[90]=\wedge_{1 \leq i \leq k} \wedge_{1 \leq j \leq|E(G)|+1, w_{i}\left(e_{j}\right)<0} \wedge_{0 \leq s \leq M}(\neg u[9, i, j-1, s] \wedge \\
& \left.\neg u\left[5, i, j,\left|w_{i}\left(e_{j}\right)\right|, s\right] \wedge \neg u[10, i, j, s]\right) \rightarrow \neg u[9, i, j, s], \\
& \varphi[91]=\wedge_{1 \leq i \leq k} \wedge_{1 \leq j \leq|E(G)|+1, w_{i}\left(e_{j}\right)<0} \wedge_{0 \leq s \leq M}(\neg u[9, i, j-1, s] \wedge \\
& \left.\neg u\left[5, i, j,\left|w_{i}\left(e_{j}\right)\right|, s\right] \wedge \neg u[10, i, j, s]\right) \rightarrow \neg u[10, i, j, s+1], \\
& \varphi[92]=\wedge_{1 \leq i \leq k} \wedge_{1 \leq j \leq|E(G)|+1, w_{i}\left(e_{j}\right)<0} \wedge_{0 \leq s \leq M}(u[9, i, j-1, s] \wedge \\
& \left.\neg u\left[5, i, j,\left|w_{i}\left(e_{j}\right)\right|, s\right] \wedge \neg u[10, i, j, s]\right) \rightarrow u[9, i, j, s], \\
& \varphi[93]=\wedge_{1 \leq i \leq k} \wedge_{1 \leq j \leq|E(G)|+1, w_{i}\left(e_{j}\right)<0} \wedge_{0 \leq s \leq M}(u[9, i, j-1, s] \wedge \\
& \left.\neg u\left[5, i, j,\left|w_{i}\left(e_{j}\right)\right|, s\right] \wedge \neg u[10, i, j, s]\right) \rightarrow \neg u[10, i, j, s+1], \\
& \varphi[94]=\wedge_{1 \leq i \leq k} \wedge_{1 \leq j \leq|E(G)|+1, w_{i}\left(e_{j}\right)<0} \wedge_{0 \leq s \leq M}(\neg u[9, i, j-1, s] \wedge \\
& \left.u\left[5, i, j,\left|w_{i}\left(e_{j}\right)\right|, s\right] \wedge \neg u[10, i, j, s]\right) \rightarrow u[9, i, j, s], \\
& \varphi[95]=\wedge_{1 \leq i \leq k} \wedge_{1 \leq j \leq|E(G)|+1, w_{i}\left(e_{j}\right)<0} \wedge_{0 \leq s \leq M}(\neg u[9, i, j-1, s] \wedge \\
& \left.u\left[5, i, j,\left|w_{i}\left(e_{j}\right)\right|, s\right] \wedge \neg u[10, i, j, s]\right) \rightarrow \neg u[10, i, j, s+1], \\
& \varphi[96]=\wedge_{1 \leq i \leq k} \wedge_{1 \leq j \leq|E(G)|+1, w_{i}\left(e_{j}\right)<0} \wedge_{0 \leq s \leq M}(\neg u[9, i, j-1, s] \wedge \\
& \left.\neg u\left[5, i, j,\left|w_{i}\left(e_{j}\right)\right|, s\right] \wedge u[10, i, j, s]\right) \rightarrow u[9, i, j, s], \\
& \varphi[97]=\wedge_{1 \leq i \leq k} \wedge_{1 \leq j \leq|E(G)|+1, w_{i}\left(e_{j}\right)<0} \wedge_{0 \leq s \leq M}(\neg u[9, i, j-1, s] \wedge
\end{aligned}
$$




$$
\begin{aligned}
& \left.\neg u\left[5, i, j,\left|w_{i}\left(e_{j}\right)\right|, s\right] \wedge u[10, i, j, s]\right) \rightarrow u[10, i, j, s+1], \\
& \varphi[98]=\wedge_{1 \leq i \leq k} \wedge_{1 \leq j \leq|E(G)|+1, w_{i}\left(e_{j}\right)<0} \wedge_{0 \leq s \leq M}(u[9, i, j-1, s] \wedge \\
& \left.u\left[5, i, j,\left|w_{i}\left(e_{j}\right)\right|, s\right] \wedge \neg u[10, i, j, s]\right) \rightarrow \neg u[9, i, j, s], \\
& \varphi[99]=\wedge_{1 \leq i \leq k} \wedge_{1 \leq j \leq|E(G)|+1, w_{i}\left(e_{j}\right)<0} \wedge_{0 \leq s \leq M}(u[9, i, j-1, s] \wedge \\
& \left.u\left[5, i, j,\left|w_{i}\left(e_{j}\right)\right|, s\right] \wedge \neg u[10, i, j, s]\right) \rightarrow u[10, i, j, s+1], \\
& \varphi[100]=\wedge_{1 \leq i \leq k} \wedge_{1 \leq j \leq|E(G)|+1, w_{i}\left(e_{j}\right)<0} \wedge_{0 \leq s \leq M}(u[9, i, j-1, s] \wedge \\
& \left.\neg u\left[5, i, j,\left|w_{i}\left(e_{j}\right)\right|, s\right] \wedge u[10, i, j, s]\right) \rightarrow \neg u[9, i, j, s], \\
& \varphi[101]=\wedge_{1 \leq i \leq k} \wedge_{1 \leq j \leq|E(G)|+1, w_{i}\left(e_{j}\right)<0} \wedge_{0 \leq s \leq M}(u[9, i, j-1, s] \wedge \\
& \left.\neg u\left[5, i, j,\left|w_{i}\left(e_{j}\right)\right|, s\right] \wedge u[10, i, j, s]\right) \rightarrow u[10, i, j, s+1], \\
& \varphi[102]=\wedge_{1 \leq i \leq k} \wedge_{1 \leq j \leq|E(G)|+1, w_{i}\left(e_{j}\right)<0} \wedge_{0 \leq s \leq M}(\neg u[9, i, j-1, s] \wedge \\
& \left.u\left[5, i, j,\left|w_{i}\left(e_{j}\right)\right|, s\right] \wedge u[10, i, j, s]\right) \rightarrow \neg u[9, i, j, s], \\
& \varphi[103]=\wedge_{1 \leq i \leq k} \wedge_{1 \leq j \leq|E(G)|+1, w_{i}\left(e_{j}\right)<0} \wedge_{0 \leq s \leq M}(\neg u[9, i, j-1, s] \wedge \\
& \left.u\left[5, i, j,\left|w_{i}\left(e_{j}\right)\right|, s\right] \wedge u[10, i, j, s]\right) \rightarrow u[10, i, j, s+1], \\
& \varphi[104]=\wedge_{1 \leq i \leq k} \wedge_{1 \leq j \leq|E(G)|+1, w_{i}\left(e_{j}\right)<0} \wedge_{0 \leq s \leq M}(u[9, i, j-1, s] \wedge \\
& \left.u\left[5, i, j,\left|w_{i}\left(e_{j}\right)\right|, s\right] \wedge u[10, i, j, s]\right) \rightarrow u[9, i, j, s], \\
& \varphi[105]=\wedge_{1 \leq i \leq k} \wedge_{1 \leq j \leq|E(G)|+1, w_{i}\left(e_{j}\right)<0} \wedge_{0 \leq s \leq M}(u[9, i, j-1, s] \wedge \\
& \left.u\left[5, i, j,\left|w_{i}\left(e_{j}\right)\right|, s\right] \wedge u[10, i, j, s]\right) \rightarrow u[10, i, j, s+1], \\
& \varphi[106]=\wedge_{1 \leq i \leq k} \wedge_{1 \leq j \leq|E(G)|+1, w_{i}\left(e_{j}\right) \geq 0} \wedge_{0 \leq s \leq M} u[9, i, j-1, s]=u[9, i, j, s], \\
& \varphi[107]=\wedge_{1 \leq i \leq k} \neg u[12, i, 0] \text {, } \\
& \varphi[108]=\wedge_{1 \leq i \leq k} \wedge_{0 \leq s \leq M}(\neg u[7, i,|E(G)|+1, s] \wedge \\
& \left.\neg d^{-}[i, s] \wedge \neg u[12, i, s]\right) \rightarrow \neg u[11, i, s], \\
& \varphi[109]=\wedge_{1 \leq i \leq k} \wedge_{0 \leq s \leq M}(\neg u[7, i,|E(G)|+1, s] \wedge \\
& \left.\neg d^{-}[i, s] \wedge \neg u[12, i, s]\right) \rightarrow \neg u[12, i, s+1], \\
& \varphi[110]=\wedge_{1 \leq i \leq k} \wedge_{0 \leq s \leq M}(u[7, i,|E(G)|+1, s] \wedge \\
& \left.\neg d^{-}[i, s] \wedge \neg u[12, i, s]\right) \rightarrow u[11, i, s], \\
& \varphi[111]=\wedge_{1 \leq i \leq k} \wedge_{0 \leq s \leq M}(u[7, i,|E(G)|+1, s] \wedge \\
& \left.\neg d^{-}[i, s] \wedge \neg u[12, i, s]\right) \rightarrow \neg u[12, i, s+1], \\
& \varphi[112]=\wedge_{1 \leq i \leq k} \wedge_{0 \leq s \leq M}(\neg u[7, i,|E(G)|+1, s] \wedge \\
& \left.d^{-}[i, s] \wedge \neg u[12, i, s]\right) \rightarrow u[11, i, s], \\
& \varphi[113]=\wedge_{1 \leq i \leq k} \wedge_{0 \leq s \leq M}(\neg u[7, i,|E(G)|+1, s] \wedge \\
& \left.d^{-}[i, s] \wedge \neg u[12, i, s]\right) \rightarrow \neg u[12, i, s+1], \\
& \varphi[114]=\wedge_{1 \leq i \leq k} \wedge_{0 \leq s \leq M}(\neg u[7, i,|E(G)|+1, s] \wedge \\
& \left.\neg d^{-}[i, s] \wedge u[12, i, s]\right) \rightarrow u[11, i, s], \\
& \varphi[115]=\wedge_{1 \leq i \leq k} \wedge_{0 \leq s \leq M}(\neg u[7, i,|E(G)|+1, s] \wedge \\
& \left.\neg d^{-}[i, s] \wedge u[12, i, s]\right) \rightarrow \neg u[12, i, s+1] \text {, }
\end{aligned}
$$




$$
\begin{aligned}
& \varphi[116]=\wedge_{1 \leq i \leq k} \wedge_{0 \leq s \leq M}(u[7, i,|E(G)|+1, s] \wedge \\
& \left.d^{-}[i, s] \wedge \neg u[12, i, s]\right) \rightarrow \neg u[11, i, s], \\
& \varphi[117]=\wedge_{1 \leq i \leq k} \wedge_{0 \leq s \leq M}(u[7, i,|E(G)|+1, s] \wedge \\
& \left.d^{-}[i, s] \wedge \neg u[12, i, s]\right) \rightarrow u[12, i, s+1], \\
& \varphi[118]=\wedge_{1 \leq i \leq k} \wedge_{0 \leq s \leq M}(u[7, i,|E(G)|+1, s] \wedge \\
& \left.\neg d^{-}[i, s] \wedge u[12, i, s]\right) \rightarrow \neg u[11, i, s], \\
& \varphi[119]=\wedge_{1 \leq i \leq k} \wedge_{0 \leq s \leq M}(u[7, i,|E(G)|+1, s] \wedge \\
& \left.\neg d^{-}[i, s] \wedge u[12, i, s]\right) \rightarrow u[12, i, s+1], \\
& \varphi[120]=\wedge_{1 \leq i \leq k} \wedge_{0 \leq s \leq M}(\neg u[7, i,|E(G)|+1, s] \wedge \\
& \left.d^{-}[i, s] \wedge u[12, i, s]\right) \rightarrow \neg u[11, i, s], \\
& \varphi[121]=\wedge_{1 \leq i \leq k} \wedge_{0 \leq s \leq M}(\neg u[7, i,|E(G)|+1, s] \wedge \\
& \left.d^{-}[i, s] \wedge u[12, i, s]\right) \rightarrow u[12, i, s+1], \\
& \varphi[122]=\wedge_{1 \leq i \leq k} \wedge_{0 \leq s \leq M}(u[7, i,|E(G)|+1, s] \wedge \\
& \left.d^{-}[i, s] \wedge u[12, i, s]\right) \rightarrow u[11, i, s], \\
& \varphi[123]=\wedge_{1 \leq i \leq k} \wedge_{0 \leq s \leq M}(u[7, i,|E(G)|+1, s] \wedge \\
& \left.d^{-}[i, s] \wedge u[12, i, s]\right) \rightarrow u[12, i, s+1], \\
& \varphi[124]=\wedge_{1 \leq i \leq k} \neg u[14, i, 0] \text {, } \\
& \varphi[125]=\wedge_{1 \leq i \leq k} \wedge_{0 \leq s \leq M}(\neg u[9, i,|E(G)|+1, s] \wedge \\
& \left.\neg d^{+}[i, s] \wedge \neg u[14, i, s]\right) \rightarrow \neg u[13, i, s], \\
& \varphi[126]=\wedge_{1 \leq i \leq k} \wedge_{0 \leq s \leq M}(\neg u[9, i,|E(G)|+1, s] \wedge \\
& \left.\neg d^{+}[i, s] \wedge \neg u[14, i, s]\right) \rightarrow \neg u[14, i, s+1], \\
& \varphi[127]=\wedge_{1 \leq i \leq k} \wedge_{0 \leq s \leq M}(u[9, i,|E(G)|+1, s] \wedge \\
& \left.\neg d^{+}[i, s] \wedge \neg u[14, i, s]\right) \rightarrow u[13, i, s], \\
& \varphi[128]=\wedge_{1 \leq i \leq k} \wedge_{0 \leq s \leq M}(u[9, i,|E(G)|+1, s] \wedge \\
& \left.\neg d^{+}[i, s] \wedge \neg u[14, i, s]\right) \rightarrow \neg u[14, i, s+1], \\
& \varphi[129]=\wedge_{1 \leq i \leq k} \wedge_{0 \leq s \leq M}(\neg u[9, i,|E(G)|+1, s] \wedge \\
& \left.d^{+}[i, s] \wedge \neg u[14, i, s]\right) \rightarrow u[13, i, s], \\
& \varphi[130]=\wedge_{1 \leq i \leq k} \wedge_{0 \leq s \leq M}(\neg u[9, i,|E(G)|+1, s] \wedge \\
& \left.d^{+}[i, s] \wedge \neg u[14, i, s]\right) \rightarrow \neg u[14, i, s+1], \\
& \varphi[131]=\wedge_{1 \leq i \leq k} \wedge_{0 \leq s \leq M}(\neg u[9, i,|E(G)|+1, s] \wedge \\
& \left.\neg d^{+}[i, s] \wedge u[14, i, s]\right) \rightarrow u[13, i, s], \\
& \varphi[132]=\wedge_{1 \leq i \leq k} \wedge_{0 \leq s \leq M}(\neg u[9, i,|E(G)|+1, s] \wedge \\
& \left.\neg d^{+}[i, s] \wedge u[14, i, s]\right) \rightarrow \neg u[14, i, s+1], \\
& \varphi[133]=\wedge_{1 \leq i \leq k} \wedge_{0 \leq s \leq M}(u[9, i,|E(G)|+1, s] \wedge \\
& \left.d^{+}[i, s] \wedge \neg u[14, i, s]\right) \rightarrow \neg u[13, i, s],
\end{aligned}
$$




$$
\begin{aligned}
\varphi[134]= & \wedge_{1 \leq i \leq k} \wedge_{0 \leq s \leq M}(u[9, i,|E(G)|+1, s] \wedge \\
& \left.d^{+}[i, s] \wedge \neg u[14, i, s]\right) \rightarrow u[14, i, s+1], \\
\varphi[135]= & \wedge_{1 \leq i \leq k} \wedge_{0 \leq s \leq M}(u[9, i,|E(G)|+1, s] \wedge \\
& \left.\neg d^{+}[i, s] \wedge u[14, i, s]\right) \rightarrow \neg u[13, i, s], \\
\varphi[136]= & \wedge_{1 \leq i \leq k} \wedge_{0 \leq s \leq M}(u[9, i,|E(G)|+1, s] \wedge \\
& \left.\neg d^{+}[i, s] \wedge u[14, i, s]\right) \rightarrow u[14, i, s+1], \\
\varphi[137]= & \wedge_{1 \leq i \leq k} \wedge_{0 \leq s \leq M}(\neg u[9, i,|E(G)|+1, s] \wedge \\
& \left.d^{+}[i, s] \wedge u[14, i, s]\right) \rightarrow \neg u[13, i, s], \\
\varphi[138]= & \wedge_{1 \leq i \leq k} \wedge_{0 \leq s \leq M}(\neg u[9, i,|E(G)|+1, s] \wedge \\
& \left.d^{+}[i, s] \wedge u[14, i, s]\right) \rightarrow u[14, i, s+1], \\
\varphi[139]= & \wedge_{1 \leq i \leq k} \wedge_{0 \leq s \leq M}(u[9, i,|E(G)|+1, s] \wedge \\
& \left.d^{+}[i, s] \wedge u[14, i, s]\right) \rightarrow u[13, i, s], \\
\varphi[140]= & \wedge_{1 \leq i \leq k} \wedge_{0 \leq s \leq M}(u[9, i,|E(G)|+1, s] \wedge \\
& \left.d^{+}[i, s] \wedge u[14, i, s]\right) \rightarrow u[14, i, s+1], \\
\varphi[141]= & \wedge_{1 \leq i \leq k} \wedge_{0 \leq s \leq M} u[11, i, s]=u[13, i, s] .
\end{aligned}
$$

Let $\xi=\wedge_{i=1}^{141} \varphi[i]$. It is not hard to check that there is a path $e_{1}, \ldots, e_{m}$ such that $p=e_{1}, q=e_{m}, \sum W\left(e_{i}\right)=D$ if and only if $\xi$ is satisfiable. Using standard transformations we can obtain an explicit transformation $\xi$ into $\zeta$ such that $\xi \Leftrightarrow \zeta$ and $\zeta$ is a $3-\mathrm{CNF}$. So, $\zeta$ gives us an explicit reduction from FDP to 3SAT. We have designed generators of natural instances for FDP. We consider our genetic algorithms OA[1] (see [22]), OA[2] (see [16]), OA[3] (see [9]), and OA[4] (see [8]) for SAT. We used heterogeneous cluster. Each test was runned on a cluster of at least 100 nodes. Selected experimental results are given in Table 1.

\begin{tabular}{|l|llll|}
\hline time & $\mathrm{OA}[1]$ & $\mathrm{OA}[2]$ & $\mathrm{OA}[3]$ & $\mathrm{OA}[4]$ \\
\hline average & $5.88 \mathrm{~h}$ & $6.21 \mathrm{~h}$ & $6.12 \mathrm{~h}$ & $5.23 \mathrm{~h}$ \\
max & $51.37 \mathrm{~h}$ & $63.38 \mathrm{~h}$ & $62.46 \mathrm{~h}$ & $44.32 \mathrm{~h}$ \\
best & $11.01 \mathrm{~min}$ & $42.33 \mathrm{~min}$ & $46.08 \mathrm{~min}$ & $14.22 \mathrm{~min}$ \\
\hline
\end{tabular}

Table 1: Experimental results for FDP.

ACKNOWLEDGEMENTS. The work was partially supported by Analytical Departmental Program "Developing the scientific potential of high school" 8.1616.2011. 


\section{References}

[1] A. Gorbenko, V. Popov, and A. Sheka, Localization on Discrete Grid Graphs, Lecture Notes in Electrical Engineering, 107 (2012), 971-978.

[2] A. Gorbenko and V. Popov, Multi-agent Path Planning, Applied Mathematical Sciences, 6 (2012), 6733-6737.

[3] A. Gorbenko and V. Popov, Programming for Modular Reconfigurable Robots, Programming and Computer Software, 38 (2012), 13-23.

[4] A. Gorbenko, A. Lutov, M. Mornev, and V. Popov, Algebras of Stepping Motor Programs, Applied Mathematical Sciences, 5 (2011), 1679-1692.

[5] A. Gorbenko, M. Mornev, V. Popov, and A. Sheka, The problem of sensor placement for triangulation-based localisation, International Journal of Automation and Control, 5 (2011), 245-253.

[6] A. Gorbenko, M. Mornev, V. Popov, and A. Sheka, The Problem of Sensor Placement, Advanced Studies in Theoretical Physics, 6 (2012), 965-967.

[7] A. Gorbenko and V. Popov, On the Problem of Sensor Placement, Advanced Studies in Theoretical Physics, 6 (2012), 1117-1120.

[8] A. Gorbenko and V. Popov, SAT Solvers for the Problem of Sensor Placement, Advanced Studies in Theoretical Physics, 6 (2012), 1235-1238.

[9] A. Gorbenko and V. Popov, Task-resource Scheduling Problem, International Journal of Automation and Computing, 9 (2012), 429-441.

[10] A. Gorbenko and V. Popov, The Binary Paint Shop Problem, Applied Mathematical Sciences, 6 (2012), 4733-4735.

[11] A. Gorbenko and V. Popov, Clustering Algorithm in Mobile Ad Hoc Networks, Advanced Studies in Theoretical Physics, 6 (2012), 1239-1242.

[12] A. Gorbenko, M. Mornev, and V. Popov, Planning a Typical Working Day for Indoor Service Robots, IAENG International Journal of Computer Science, 38 (2011), 176-182.

[13] A. Gorbenko and V. Popov, Self-Learning Algorithm for Visual Recognition and Object Categorization for Autonomous Mobile Robots, Lecture Notes in Electrical Engineering, 107 (2012), 1289-1295.

[14] A. Gorbenko and V. Popov, Robot Self-Awareness: Usage of Co-training for Distance Functions for Sequences of Images, Advanced Studies in Theoretical Physics, 6 (2012), 1243-1246. 
[15] A. Gorbenko and V. Popov, Robot's Actions and Automatic Generation of Distance Functions for Sequences of Images, Advanced Studies in Theoretical Physics, 6 (2012), 1247-1251.

[16] A. Gorbenko and V. Popov, Computational Experiments for the Problem of Selection of a Minimal Set of Visual Landmarks, Applied Mathematical Sciences, 6 (2012), 5775-5780.

[17] A. Gorbenko and V. Popov, The Problem of Selection of a Minimal Set of Visual Landmarks, Applied Mathematical Sciences, 6 (2012), 4729-4732.

[18] A. Gorbenko and V. Popov, Face Detection and Visual Landmarks Approach to Monitoring of the Environment, International Journal of Mathematical Analysis, 7 (2013), 213-217.

[19] V. Popov, Partially Distinguishable Guards, Applied Mathematical Sciences, 6 (2012), 6587-6591.

[20] A. Gorbenko and V. Popov, The Problem of Selection of a Set of Partially Distinguishable Guards, Applied Mathematical Sciences, 7 (2013), 651654 .

[21] A. Gorbenko and V. Popov, A Real-World Experiments Setup for Investigations of the Problem of Visual Landmarks Selection for Mobile Robots, Applied Mathematical Sciences, 6 (2012), 4767-4771.

[22] A. Gorbenko and V. Popov, On the Problem of Placement of Visual Landmarks, Applied Mathematical Sciences, 6 (2012), 689-696.

[23] A. Gorbenko, V. Popov, and A. Sheka, Robot Self-Awareness: Temporal Relation Based Data Mining, Engineering Letters, 19 (2011), 169-178.

[24] A. Gorbenko and V. Popov, Robot Self-Awareness: Occam's Razor for Fluents, International Journal of Mathematical Analysis, 6 (2012), 14531455 .

[25] A. Gorbenko, V. Popov, and A. Sheka, Robot Self-Awareness: Exploration of Internal States, Applied Mathematical Sciences, 6 (2012), 675688.

[26] A. Gorbenko and V. Popov, The c-Fragment Longest Arc-Preserving Common Subsequence Problem, IAENG International Journal of Computer Science, 39 (2012), 231-238.

[27] A. Gorbenko and V. Popov, Robot Self-Awareness: Formulation of Hypotheses Based on the Discovered Regularities, Applied Mathematical Sciences, 6 (2012), 6583-6585. 
[28] A. Gorbenko and V. Popov, Anticipation in Simple Robot Navigation and Learning of Effects of Robot's Actions and Changes of the Environment, International Journal of Mathematical Analysis, 6 (2012), 2747-2751.

[29] A. Gorbenko and V. Popov, Anticipation in Simple Robot Navigation and Finding Regularities, Applied Mathematical Sciences, 6 (2012), 6577-6581.

[30] A. Gorbenko and V. Popov, The Force Law Design of Artificial Physics Optimization for Robot Anticipation of Motion, Advanced Studies in Theoretical Physics, 6 (2012), 625-628.

[31] S. Kagami, T. Kitagawa, K. Nishiwaki, T. Sugihara, M. Inaba, and H. Inoue, A Fast Dynamically Equilibrated Walking Trajectory Generation Method of Humanoid Robot, Autonomous Robots, 12 (2002), 71-82.

[32] A. Gorbenko and V. Popov, On Face Detection from Compressed Video Streams, Applied Mathematical Sciences, 6 (2012), 4763-4766.

[33] A. Gorbenko and V. Popov, Usage of the Laplace Transform as a Basic Algorithm of Railroad Tracks Recognition, International Journal of Mathematical Analysis, 6 (2012), 2413-2417.

[34] A. Gorbenko and V. Popov, An Intelligent Gradient Detector for Monitoring of Passenger Flows, International Journal of Mathematical Analysis, 7 (2013), 637-641.

[35] J. J. Kuffner, S. Kagami, K. Nishiwaki, M. Inaba, and H. Inoue, Dynamically-stable motion planning for humanoid robots, Autonomous Robots, 12 (2002), 105-118.

[36] A. Gorbenko and V. Popov, Footstep Planning for Humanoid Robots, Applied Mathematical Sciences, 6 (2012), 6567-6571.

[37] N. Perrin, Footstep Planning for Humanoid Robots: Discrete and Continuous Approaches. PhD Dissertation, Institut National Polytechnique de Toulouse, 2011.

[38] A. Gorbenko and V. Popov, The Minimum Test Collection Problem, Applied Mathematical Sciences, 7 (2013), 1191-1193.

[39] A. Gorbenko and V. Popov, The Farthest Substring Problem, Applied Mathematical Sciences, 7 (2013), 1209-1212.

[40] A. Gorbenko and V. Popov, Computational Experiments for the Problem of Hamiltonian Path with Fixed Number of Color Repetitions, Advanced Studies in Theoretical Physics, 7 (2013), 121-126. 
[41] A. Gorbenko and V. Popov, On Hamilton Paths in Grid Graphs, Advanced Studies in Theoretical Physics, 7 (2013), 127-130.

[42] A. Gorbenko and V. Popov, Computational Experiments for the Problem of Sensor-Mission Assignment in Wireless Sensor Networks, Advanced Studies in Theoretical Physics, 7 (2013), 135-139.

[43] A. Gorbenko and V. Popov, The Swap Common Superstring Problem, Applied Mathematical Sciences, 7 (2013), 609-614.

[44] A. Gorbenko and V. Popov, The String Barcoding Problem, Applied Mathematical Sciences, 7 (2013), 615-622.

[45] A. Gorbenko and V. Popov, On Multiple Occurrences Shortest Common Superstring Problem, Applied Mathematical Sciences, 7 (2013), 641-644.

[46] A. Gorbenko and V. Popov, Longest Common Parameterized Subsequences with Fixed Common Substring, Applied Mathematical Sciences, 7 (2013), 645-650.

[47] A. Gorbenko and V. Popov, The Hamiltonian Alternating Path Problem, IAENG International Journal of Applied Mathematics, 42 (2012), 204213.

[48] A. Gorbenko and V. Popov, The Problem of Finding Two Edge-Disjoint Hamiltonian Cycles, Applied Mathematical Sciences, 6 (2012), 6563-6566.

[49] A. Gorbenko and V. Popov, Multiple Occurrences Shortest Common Superstring Problem, Applied Mathematical Sciences, 6 (2012), 6573-6576.

[50] A. Gorbenko and V. Popov, The Far From Most String Problem, Applied Mathematical Sciences, 6 (2012), 6719-6724.

[51] A. Gorbenko and V. Popov, Hamiltonian Alternating Cycles with Fixed Number of Color Appearances, Applied Mathematical Sciences, 6 (2012), 6729-6731.

[52] A. Gorbenko and V. Popov, On the Longest Common Subsequence Problem, Applied Mathematical Sciences, 6 (2012), 5781-5787.

[53] A. Gorbenko and V. Popov, The Longest Common Parameterized Subsequence Problem, Applied Mathematical Sciences 6 (2012), 2851-2855.

[54] A. Gorbenko and V. Popov, The set of parameterized k-covers problem, Theoretical Computer Science, 423 (2012), 19-24. 
[55] M. Nykänen and E. Ukkonen, Finding paths with the right cost, in Proceedings of the 16th Conference on Theoretical Aspects of Computer Science, 1999, 345-355.

[56] C. H. Papadimitriou, On the Complexity of Integer Programming, Journal of the Assoclauon for Compuung Machinery, 28 (1981), 765-768.

\section{Received: February 12, 2013}

